\title{
Captura de Rhynchophorus palmarum (L.) pelo Uso de Feromônio de Agregação Associado a Árvore-Armadilha e Inseticida
}

\author{
José I. L. Moura ${ }^{1}$ José M. S. Bento ${ }^{2}$, Jonas de Souza ${ }^{1}$ e Evaldo F. Vilela ${ }^{3}$ \\ ${ }^{1}$ Estação Experimental Lemos Maia, CEPLAC, 45690-000, Una, BA. \\ ${ }^{2}$ IÍCA/EMBRAPA/CNPMF, Caixa postal 07, 44380-000, Cruz das Almas, BA. \\ ${ }^{3}$ Universidade Federal de Viçosa, Departamento de Biologia Animal, 36571-000, \\ Viçosa, MG.
}

An. Soc. Entomol. Brasil 26(1): 69-73 (1997)

Field Trapping of Rhynchophorus palmarum (L.) Using Trap Plants Treated with Aggregation Pheromone and Insecticide

\begin{abstract}
The efficacy of the aggregation pheromone of Rhynchosphorus palmarum (L.), 2(E)-6-methyl-2-hepten-4-ol, mixed with insecticide to capture adults of this pest on oil palm tree Elaeis guineensis was evaluated. Cuttings into the trunk of oil palm trees attacked by Rhadinaphelenchus cocophilus Cobb., the agent of red ring disease, were sprayed with Furadan 350 SC plus 3 $\mathrm{ml}$ 2(E)-6-methyl-2-hepten-4-ol. Another group of plants was treated with insecticide only. The capture of $R$. palmarum on oil palm trees treated with pheromone plus insecticide was 65 to $89 \%$ greater than those treated with insecticide only.
\end{abstract}

KEY WORDS: Insecta, Coleoptera, Curculionidae, palm weevil, Elaeis guineensis, red ring disease.

RESUMO - A eficiência do feromônio de agregação do Rhynchophorus palmaram (L.), 2(E)-6-methyl-2-hepten-4-ol, associado com inseticida foi avaliada na captura de adultos desta praga em árvores de dendenzeiros Elaeis guineensis. Entalhes confeccionados em árvores de dendenzeiros atacadas pelo nematóide Rhadinaphelenchus cocophilus Cobb., agente causal da doença do anel-vermelho foram pulverizados com inseticida (Furadan $350 \mathrm{SC}$ ) mais $3 \mathrm{ml}$ do feromônio. Outro grupo de árvores foram tratadas somente com inseticida. A captura de $R$. palmarum nas árvores tratadas com feromônio mais inseticida foi entre 65 e $89 \%$ superior ao tratamento com inseticida.

PALAVRAS-CHAVE: Insecta, Coleoptera, Curculionidae, bicudo das palmá-ceas, Elaeis guineensis, anel-vermelho.

$\mathrm{O}$ adulto de Rhynchophorus palmarum (L.) (Coleoptera: Curculionidae) é o principal vetor do nematóide Rhadinaphelenchus cocophilus Cobb., agente causal da doença do anel-vermelho (Genty et al. 1978). Esta doença é mortal para coqueiros (Cocos nucifera), dendenzeiros (Elaeis guineensis) e outras palmeiras.
Plantas debilitadas pelo anel-vermelho ou que tenham sofrido ferimento mecânico ou ata-que por inseto, exalam odores de fermentação da seiva que atraem os adultos de $R$. palmarum (Bondar 1940, Moura et al. 1989, Rochat 1987). Uma vez encontrada a planta hospedeira, o macho de $R$. palmarum 
libera um feromônio de agregação atraindo fêmeas e outros machos, que se agrupam sobre à fonte de alimento, promovendo o acasalamento (Nadarajan 1986, Rochat 1987, Moura et al. 1989, Sanches et al. 1993). Ao se alimentarem de plantas debilitadas pelo anel-vermelho, estes insetos tornam-se vetares do nematóide $R$. cocophilus reiniciando o ciclo da doença.

O controle de $R$. palmarum tem sido baseado no uso de atrativos alimentares, principalmente iscas a base de estipe de dendenzeiros e coqueiros ou toletes de cana-de-açúcar colocadas em diversos tipos de armadilhas para capturar e matar os adultos (Morin et al. 1988, Chinchil et al. 1990, Moura et al. 1991).

Com a descoberta do feromônio de agregação do $R$. palmarum, o 2(E)-6-methyl-2hepten-4-ol (Rochat et al. 1991), esperava-se uma redução no uso de atrativos alimentares. Entretanto, demonstrou-se que o feromônio exercia pouca ou nenhuma atratividade sobre os demais adultos quando utilizado sozinho, havendo a necessidade de associá-lo a um atrativo alimentar o que o tornava eficiente (Oehlschlager et al. 1992, Jaffe et al. 1993). Isto fez com que as técnicas de manejo visando o controle desta praga fossem aprimoradas, recomendando-se a associação do feromônio mais a presença de um atrativo alimentar. $\mathrm{O}$ uso de árvore-armadilha, que consiste no aproveitamento de plantas atacadas pelo anelvermelho impregnadas com inseticida mais feromônio, ainda não foi avaliado como método de controle de $R$. palmarum, sendo este o objetivo deste trabalho.

\section{Material e Métodos}

Utilizou-se para este estudo um plantio, de 33 ha de dendenzeiros, com idade de 15 anos, na Estação Experimental Lemos Maia (CEPLAC), em Una-BA, durante os meses de julho a outubro de 1995.

Foram selecionadas 22 árvores atacadas na fase inicial da doença anel-vermelho, como atrativo alimentar aos adultos de $R$. palmarum. Nestas plantas, promoveu-se no tronco, a uma altura de $1,5 \mathrm{~m}$ um entalhe no formato de cunha de 15 a $20 \mathrm{~cm}$ de profundidade. Em seguida, preparou-se uma calda inseticida, misturando-se $120 \mathrm{ml}$ de Furadan $350 \mathrm{SC}$ em 201 de água, sendo utilizados cerca de $50 \mathrm{ml}$ da calda em cada entalhe. Abaixo do entalhe, efetuou-se um corte transversal e injetou-se com seringa, aproximadamente, $100 \mathrm{ml}$ do herbicida Daconate (MSMA), com a finalidade de acelerar a mortalidade dos dendenzeiros, aumentando a atratividade destas plantas para adultos de $R$. palmarum. Ao acaso, selecionou-se 11 das 22 árvores para receberem $3 \mathrm{ml}$ do feromônio de agregação 2(E)-6-methyl-2hepten-4-ol, dentro dos entalhes. Este feromônio foi acondicionado em tubos de plástico contendo microporos, para liberação lenta do produto. Assim, permitiu-se que os adultos de $R$. palmarum atraídos pelos odores liberados dos entalhes das plantas com ou sem feromônio fossem mortos em contato com o inseticida.

As avaliações consistiram da retirada e contagem dos adultos de $R$. palmarum capturados nos entalhes a cada 10 dias. Após 120 dias, os dendenzeiros foram seccionados transversalmente visando constatar se algum inseto havia escapado ou não da ação do inseticida e colonizado o interior do tronco.

\section{Resultados e Discussão}

Ao longo de 120 dias foram coletados 1089 adultos do $R$. palmarum nas árvores-armadilha contendo o feromônio de agregação, contra 330 nas árvores-armadilha sem o feromônio. Em todas as avaliações a cada 10 dias, o tratamento com o feromônio correspondeu entre 65 e $89 \%$ de todos os adultos do $R$. palmarum capturados no período (Fig. 1).

As árvores-armadilha associadas ao feromônio permanecem mais tempo atrativas ao $R$. palmarum do que iscas a base de cana-deaçúcar ou pedaços de estipe de palmáceas. Segundo Moura et al. (1991), o tempo de duração da atração das iscas com cana-de-açúcar e pedaços de estipe de palmáceas é de, aproxi- 


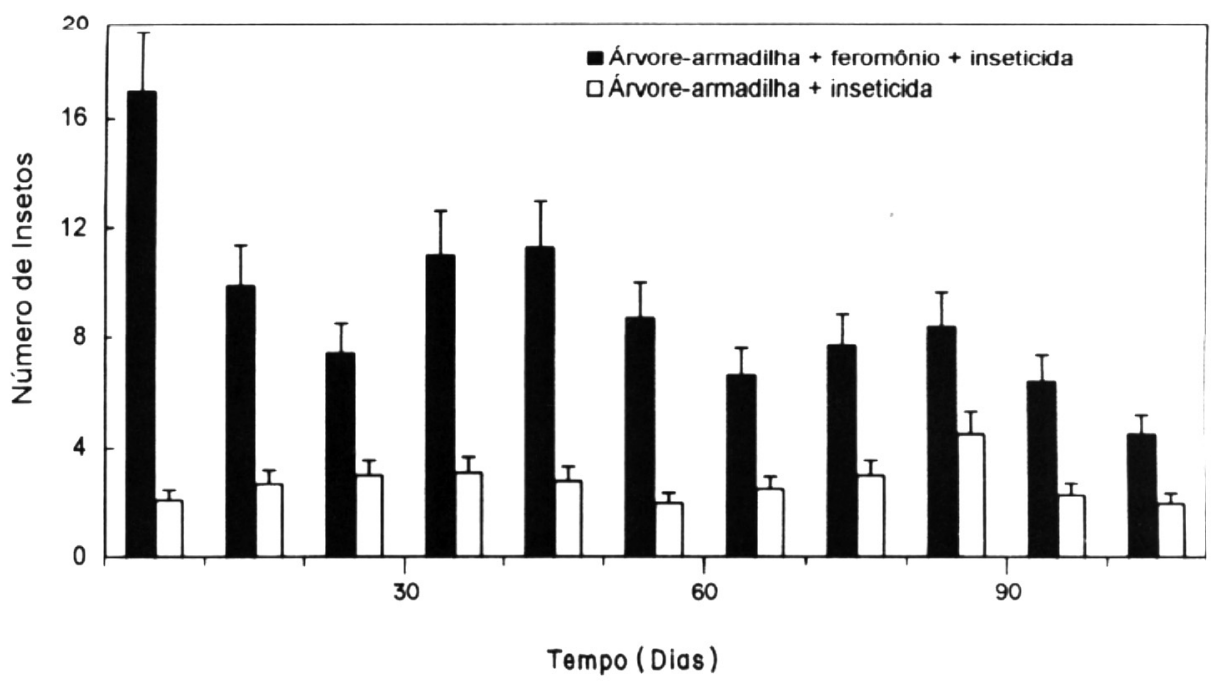

Figura 1. Média ( \pm EP) do número de Rhynchophorus palmarum L. capturados a cada 10 dias em plantação de dendenzeiro Elaeis guineensis contendo 11 árvores-armadilha + feromônio + inseticida e 11 árvores-armadilha + inseticida ao longo de 120 dias entre julho e outubro de 1995, em Una, BA.

madamente, 15 e sete dias, respectivamente. No caso das árvores-armadilha, constatou-se que as mesmas se mantiveram atrativas ao longo de três meses. Embora estas plantas possivelmente viessem a atrair novos adultos de $R$. palmarum, $o$ seu uso não é aconselhado. Após este período, corre-se o risco de alguns insetos escaparem a ação do inseticida e colonizarem o interior do meristema do dendenzeiro, já que o ciclo de vida deste inseto é cerca de quatro meses e o herbicida não tem ação sobre as formas jovens. Das 22 plantas seccionadas trans- versalmente, somente duas apresentaram larvas de $R$. palmarum nos entalhes. Entretanto, um corte longitudinal nos dendenzeiros demonstrou que oito árvores possuíam uma ou mais larvas. Com isso, devese eliminar estas árvores após três meses de uso como árvores-armadilha.

A vantagem no uso de árvores-armadilha com feromônio, impregnadas com inseticida, está no aproveitamento dos dendenzeiros atacados pelo anel-vermelho e na sua eficiência na coleta dos adultos de $R$. palmarum. Tratando-se de plantações acima de 10 anos é mais viável economicamente que a simples erradicação das árvores atacadas ou, possivelmente, que o uso de outras armadilhas a base de cana-de-açúcar ou estipe de palmáceas (Moura et al. 1995).

A desvantagem deste método é de não poder ser utilizado em plantações com menos de 10 anos devido a espessura das árvores. Outra desvantagem está na constante exposição da doença no campo, pois pode ocorrer que um besouro se alimente da planta sem entrar em contato com o inseticida e infecte outras palmas, ou ainda, de uma forma mais remota, que outros insetos ou instrumentos cortantes, transmitam a doença (Franco 1964, Dean 1979). Outra possibilidade, seria a contaminação de uma planta doente para uma planta sadia via sistema radicular (Warwick \& Bezerra 1992).

Num programa de manejo integrado de dendenzeiro em Nazaré, BA, Moura et al. 
(1995) reduziram, ao longo de dois anos, em até $65 \%$ a população de $R$. palmarum e em $8 \%$ a doença do anel-vermelho, numa área de 1.115 ha. Dentre as táticas empregadas, citase o uso de árvores-armadilha com inseticida mais herbicida. Contudo, quando as plantas se apresentavam secas pela ação do herbicida, utilizou-se fogo com o propósito de eliminar os insetos nela presente. Neste caso, observou-se larvas, pupas e adultos de $R$. palmarum mortos pela ação do fogo.

Em plantações de dendê no Norte do Brasil, onde casos de anel-vermelho estão associados com o amarelecimento fatal, doença letal ainda desconhecida, o uso de árvore-armadilha com feromônio poderá ser bem sucedido, pois nestas plantações os dendenzeiros não são erradicados, e sim mortos através da aplicação de óleo diesel na região da flecha (J.I.L. Moura, não publicado).

Observou-se, ainda, que Rhinostomus barbirostris (L.) foi atraído pelas árvores-armadilha com e sem feromônio. Este curculionideo, como $R$. palmarum, é uma séria praga dos coqueiros do Nordeste do Brasil. Entretanto, não é atraído por iscas constituídas de estipe de palmáceas e cana-de-açúcar. Desse modo, esta técnica poderá ser útil em estudos etológicos e de controle do R. barbirostris.

\section{Literatura Citada}

Bondar, G. 1940. Insetos nocivos e moléstias do coqueiro (Cocos mucifera L.) no Brasil. Salvador: Tipografia Naval, 156p.

\section{Chinchilia, G., R. Menjivar \& E. Árias. 1990.} Picudo de Ia paloma y efermedade dei Anillo Roja/Hoja pequena en uma plantación comercial em Honduras. Turrialba 40:471-477.

Dean, C.G. 1979. Red ring disease ö̈Cocus mucifera L. caused by Rhadina-phelenchus cocophilus (Cobb, 1919) Godoy,
1960. An annoted bibliography and Review. St. Albans, Herts, Commonwaith Institute of Helmin- thology. Tech. Com. 1 47, 70p.

Franco, E. 1964. Estudo sobre o anel vermelho do coqueiro. Sergipe. Insp. Def. San.Veg., 235p.

Genty, Ph., R. D. de Chenon, J. P. Morin \& C. A. Korytkowski. 1978. Oléagineux, Reseul inter dês corpos gras. Oléagineux 33: 326-419.

Jaffé, K., P. Sanches, H. Cerda, J. V. Hernandez, R. Jaffé, N. Urdaneta, G. Guerra, R. Martinez \& B. Miras. 1993. Chemical ecology the palm weevil Rhynchophorus palmarum (L.) (Coleoptera: Curculionidae): Attraction to host plants and to a male-produced aggregation pheromone. J. Chem. Ecol. 19:17031720 .

Morin, J.R, F. Lucchini, J.C.A.Araújo, J.M.S. Ferreira \& L.S. Fraga. 1988. Lê controle de Rhynchophorus palmarum piegeage à 1 'aide de morceaux de palmier. Oléagineux 41: 57-62.

Moura, J.LL., R. Sgrillo,E.F. Vilela, M.A.G. Aguilar \& M.L.V. de Rezende. 1989. Estudos do compotamento olfativo de Rhynchophorus palmarum (L.) (Coleoptera: Curculionidae) no campo. An. Soc. Entomol. Brasil 18: 267-273.

Moura, J. I. L., M. L. V. de Rezende, M. F. de Lima \& D. L. Q. Santana. 1991. Táticas para o controle integrado de Rhynchophorus palmarum (L.). Ilhéus, CEPLAC/ CEPEC,16p.

Moura, J.LL., M.L.B. de Rezende \& E.F. Vilela. 1995. Manejo integrado do Rhyn- 
chophorus palmarum (L.) (Coleoptera: Curculionidae) em plantios de dendê na Bahia. An. Soc. Entomol. Brasil 24: 501506.

Nadarajan, L. 1986. Investigation on the pheromonal comunication in the palm weevil Rhynchophorus palmarum L. (Coleoptera: Curculionidae). Paris, France: INRA, $38 \mathrm{p}$.

Oehlschlager,A. C., H. D. Pierce, B. Morgan, P. D. C. Wimalaratne, K. N. Slessor, G. G. S. King, G. Gries, R. Gries, J. H. Borden, L. F. Jiron, C. M. Chinchilia \& R. Mexzon, R. 1992. Chirality and field testing of rhynchophorol, the aggregation pheromone of the american palm weevil Rhynchophorus palmarum (L.). Naturwissenschaften79: 134-135.

Rochat, D. 1987. Estude de Ia comunication chimiques chez un coleoptere curculio nidae: Rhynchophorus palmarum L. (Co- leoptera: Curculionidae). Mariverciste Paris VI Institut National Agronomique. Paris-Grinon. 30p.

Rochat, D., A. Gonzales, D. Mariau, A. Vilianueva \& P. Zagztti. 1991. Evidence for male-produced aggregation pheromone in american palm weevil, Rhynchophoruspalmarum. J. Chem. Ecol. 17:12211230.

Sanches, P.A., K. Jaffé, J. V. Hernandez \& H. Creda. 1993. Biologia y comportamento dei picudo dei cocotero Rhynchophorus palmarum L. (Coleoptera: Curculionidae). Bol. Entomol. Venez. 8: 82-93.

Warwick, D.R.N. \& A.P.T. Bezerra. 1992. Possible root transmission of the pod ring nematode (Rhadinaphelenchus cocophilus) to coconnut palms. Plant Disease 2: 809-811.

Recebido em 30/05/96. Aceito em 22/01/97. 